\title{
edmetic
}

Revista de Educación Mediática y TIC

\section{Presentación: Aplicaciones de la Realidad Aumentada en educación}

Hablar de "Realidad Aumentada" ("Augmented Reality") (RA) es posiblemente centrarnos en una de las tecnologías de las denominadas emergentes que con más fuerza se están acercando a diferentes terrenos de la sociedad, que van desde el ocio, la publicidad, el marketing y por supuesto en el terreno educativo como está siendo puesto de manifiesto tanto desde diferentes Informes Horizon (García et al., 2010; Johnson et al., 2016), como en los Reportes EduTrend del Observatorio del Tecnológico de Monterrey (Tecnológico de Monterrey, 2015), o en los hiperciclos tecnológicos que elabora anualmente la compañía "Gardner Research" (http://www.gartner.com/technology/home.jsp), líder mundial en investigación y asesoramiento en Tecnologías de la Información y Comunicación (TIC).

Tecnología de la RA que permite combinar en tiempo real información digital e información física a través de diferentes soportes tecnológicos como por ejemplo las Tablets o Smartphones, para crear de esta forma una nueva realidad enriquecida. Su significación para la formación viene determinada, por una parte por sus características específicas, como son: ser una realidad mixta, la posibilidad que ofrece para integrar en tipo real tanto diferentes capas de información como información en diferentes tipos de formatos (textos, url, vídeos,...), es una tecnología interactiva, su facilidad de manejo, y que mediante su utilización enriquecemos o alteramos la información de la realidad añadiéndole información adicional (Cabero y García, 2016; Cabero y Barroso, 2016); y por otra, porque los dispositivos que se suelen utilizar para su observación, como son los Smartphone, son tecnologías de las que disponen 
con facilidad.

Ahora bien, sobre estas posibilidades también nos encontramos con diferentes inconvenientes y limitaciones, para su incorporación en la enseñanza, que van desde la novedad de la tecnología y por tanto la falta de experiencias educativas, la carencia de marcos conceptuales que aporten bases para la toma de decisiones para su incorporación a los procesos de enseñanza aprendizaje, la falta de investigaciones educativas y la falta de publicaciones científicas y académicas.

Son algunos de estos los motivos que ha llevado a la revista "Edmetic. Revista de Educación mediática y TIC" a la organización de este monográfico sobre las posibilidades educativas que ofrece esta tecnología.

El monográfico se ha articulado alrededor de nueve artículos, que recogen experiencias de utilización de la RA, tanto en diferentes niveles y contextos educativos, como de diferentes países y comunidades autonómicas españolas.

El primer artículo "Realidad Aumentada: Una revolución educativa" elaborado por G Iban de la Horra desde el colegio Divina Providencia (Valladolid,), nos adentra en el mundo de la RA desde la perspectiva educativa, la cual acerca al lector a un gran número de aplicaciones, las cuales permiten al educador introducir los contenidos de manera más lúdica y efectiva. Todo ello desde un punto de vista crítico y reflexivo se hacen latentes las bondades de esta tecnología en los diversos niveles educativos.

La segunda aportación títulada "Producción de recursos de aprendizaje apoyados en realidad aumentada por parte de los estudiantes de magisterio", realizado en la Facultad de Educación y en el Secretariado de Recursos Audiovisuales y Nuevas Tecnologías de la Universidad de Sevilla, y elaborado por Julio Barroso Osuna y Oscar Gallego Pérez, nos ofrece los resultados alcanzados con estudiantes universitarios donde éstos se convierten en productores de objetos de aprendizaje en RA. El artículo nos sugiere nuevas posibilidades de incorporar esta tecnología a la práctica educativa, y es aquella donde los alumnos se convierten en proconsumidores de esta tecnología.

El tercer artículo ("La producción científica sobre Realidad Aumentada, edmetic, 6(1), 2017, E-ISSN: 2254-0059; pp. 4-8 
un análisis de la situación educativa desde la perspectiva Scopus") ha sido realizado los profesores Fombona y Pascual de la Universidad de Oviedo, nos ofrecen una excelente revisión de artículos científicos publicados sobre la utilización educativa de esta tecnología. En el artículo se analiza la evolución y el estado actual de la RA. Es significativo las conclusiones a las que se llegan y también la revisión bibliográfica que nos aportan para la profundización en la temática de la RA.

El artículo elaborado por Javier Sánchez, "El potencial de la realidad aumentada en la enseñanza de español como lengua extranjera", nos describe las posibilidades que esta tecnología nos ofrece para crear escenarios educativos que faciliten el aprendizaje del español como segunda lengua extranjera. En el artículo se parte de la definición de la RA, se presentan los diferentes niveles con que nos podemos encontrar de ella, y las posibilidades educativas que nos ofrece esta tecnología. Se presenta una excelente revisión de experiencias educativas.

En el quinto artículo, El artículo "Experiencias formativas de uso didáctico de la realidad aumentada con alumnado del grado de educación primaria en la Universidad de Málaga", elaborado por Noelia M. Moreno Martínez y Juan Leíva de la Universidad de Málaga, nos presenta la experiencia llevada a cabo en el Grado de Educación Primaria de la Universidad de Málaga durante el curso académico 2015-2016, y que persigue como objetivo la explicación y presentación de las principales herramientas de la RA para dispositivos móviles y ordenadores. En el artículo se analizan las actitudes que despiertan en los estudiantes y la adquisición de competencias de uso de estos recursos desde un punto de vista didáctico para su implementación con un carácter prospectivo en las instituciones educativas en las que ejercerán su labor docente.

Sergio Álvarez Sánchez, Laura Delgado Martín, Miguel Ángel Gimeno González, Teresa Martín García, Fernando Almaraz Menéndez y Camilo Ruiz Méndez, firman la sexta aportación denominada "El Arenero Educativo: La Realidad Aumentada un nuevo recurso para la enseñanza", y describe la experiencia realizada en la Universidad de Salamanca para implementar un recurso para la enseñanza de las Matemáticas y las Ciencias Naturales, mediante la instalación de una cámara infrarroja para leer la superficie 
tridimensional de la arena y después dibujar sobre ella curvas de nivel y cuerpos de agua que se transforman cuando el usuario interactúa con la arena. La experiencia se lleva a cabo en las etapas de la Educación Secundaria Obligatoria y Bachillerato, y aporta ideas sobre las posibilidades educativas de esta tecnología para crear entornos interactivos formativos.

La perspectiva internacional la aporta la visión de $M^{a}$ Magdalena Madrigal Lozano, Laura A. Hernández Moreno, Juan G. López Solórzano y Alma E. Merla González, quienes a través de la propuesta Incursión de tecnologías emergentes en una escuela pública de negocios de México

El artículo "Percepciones del alumnado hacia el aprendizaje mediante objetos educativos enriquecidos con realidad aumentada" realizado por Urtza Garay Ruíz, Eneko Tejada Garitano y Carlos Castaño Garrido de la Universidad del País Vasco, nos presenta también una investigación apoyada en el modelo TAM de Davis para conocer el grado de aceptación que esta tecnología despierta en los alumnos universitarios. Conjuntamente con el primero del monográfico, nos permiten ir elaborando una teoría sobre la significación que esta tecnología tiene para los alumnos universitarios.

Cierra el monográfico la propuesta titulada Realidad Aumentada, proyectos en el aula de primaria: experiencias y casos en Ciencias Sociales y firmada por Ramón Cozar Gutiérrez y José Manuel Sáez López

\section{Referencias bibliográficas}

CABERO, J., Y BARROSO, J. (2016). The educational possibilities of Augmented Reality. NAER, Journal of New Approaches in Educational Research, 5(1), 44-50. doi: 10.7821/naer.2016.1.140

CABERO, J., y GARCÍA, F. (coords.) (2016). Realidad aumentada. Tecnología para la formación. Madrid: Síntesis.

GARCíA, I., PEÑA, I., JHONSON, L., SMITH, R., LEVINE, A., y HAYWOOD, K. (2010). Informe Horizon: Edición Iberoamericana 2010. Austin, Texas: The New Media Consortium.

JOHNSON, L., ADAMS BECKER, S., CUMMINS, M., ESTRADA, V., FREEMAN, A., Y HALL, C. (2016). NMC Horizon Report: 2016 Higher Education Edition. Austin, Texas: The New Media Consortium.

edmetic, 6(1), 2017, E-ISSN: 2254-0059; pp. 4-8

(c) edmetic, Revista de Educación Mediática y TIC 
Julio Cabero Almenara

TECNOLÓGICO DE MONTERREY (2015). Reporte EduTrends. Radar de Innovación Educativa 2015. Monterrey: Tecnológico de Monterrey.

Dr. Julio Cabero Almenara

Universidad de Sevilla

cabero@us.es 